\title{
Reflections on Study Strategy Modifications Using Cognitive Load Theory and Dual Processing Theory in the First Year of Medical School
}

\author{
Caroline Zhao ${ }^{1}$ Yenya $\mathrm{Hu}^{2}$ \\ Accepted: 22 December 2020 / Published online: 22 January 2021 \\ (C) International Association of Medical Science Educators 2021
}

\begin{abstract}
Navigating the beginning of medical school can be challenging for students. The new learning environment and the increased volume and complexity of information presented within a short period of time demand more efficient and effective study strategies. We describe the experience and perspective of a first-year medical student, as well as the adjustment of study strategies based on the application of cognitive load theory and dual processing theory. We provide practical approaches that promote intentional and individualized studying planning and better knowledge retention, which may also improve the wellbeing of the new medical students.
\end{abstract}

Keywords First-year medical student $\cdot$ Study strategy adjustment $\cdot$ Cognitive load $\cdot$ Dual processing theory

\section{Introduction}

The transition to medical school and navigating its intense academic demands can be challenging for many students. First-year students often find it hard to adapt to the significantly increased volume of information presented in an often shortened preclinical curriculum, as well as the accompanying studying demands, regardless of previous preparation [1]. This process may be even more difficult for non-traditional students who have spent time in the workforce and must readjust to classroom learning [2-4].

At the beginning of medical school, it is natural for students to utilize study strategies from college because they worked before. For some students, there is no need to change these habits because they continue to be effective; however, for others, this is not the case. Many students utilize memorization of lecture notes as a main tool for studying in college, be it through flashcards, rewriting notes, or rote fact memorization. When there are no effective approaches in place to handle the

Yenya $\mathrm{Hu}$

yehu@wakehealth.edu

1 Feinberg School of Medicine, Northwestern University, Chicago, IL, USA

2 Medical Education, Wake Forest School of Medicine, Winston-Salem, NC, USA fast-paced medical school curriculum, students may resort back to these previously effective methods [5].

In college, individual courses rarely require significant retention for integration purposes; thus, memorization may be effective. However, in medical school, the integration of basic knowledge into system-based learning is essential to understanding disease pathophysiology. For example, when learning about cystic fibrosis during the pulmonary system, students need to integrate their knowledge of the disciplines of cell biology and genetics, coupled with that of the gastrointestinal and reproductive systems, in order to have an in-depth understanding of the disease's pathophysiology. Medical school curriculums are designed to build and weave a knowledge base that needs to be maintained in order to have a deep understanding of the diseased state. This level of knowledge integration is required both on the board examinations and clinically in the students' future careers as physicians [6]. However, because of the volume and intensity of the curriculum, the need for knowledge retention and intentional integration can often go unrecognized or underappreciated during the preclinical years.

This type of experience greatly resonated my (first author, $\mathrm{CZ}$ ) experience during the first year of medical school. In this monograph, I reflect on my first year, the pitfalls I encountered, and how I worked with my advisor (corresponding author, $\mathrm{YH}$ ) to adjust my study strategies to make the experience less stressful, more enjoyable, and more effective. 


\section{First-year Curriculum and Assessment in Feinberg School of Medicine}

While the first author attends Northwestern, the study approaches described in this paper can be applied to any integrated or traditional curriculum. The first organizing principle of the Northwestern Feinberg School of Medicine's curriculum is integration. It integrates four elements, Science in Medicine, Clinical Medicine, Health and Society, and Professional Development. The curriculum also contains five threads that are woven throughout all four years of medical school: medical decision-making and diagnostic testing, health care quality and patient safety, teamwork and leadership, lifestyle medicine, and health advocacy and equity [7].

The Science in Medicine element, which is especially emphasized in the preclinical years, is designed to integrate basic science topics into systems. First, the general principles are covered in Block 1, made up of three Foundations modules. Foundations 1 covers biochemistry, cell biology, and genetics. Foundations 2 covers pharmacology, embryology, anatomy, histology, and pathology and has a continuation of genetics. Foundations 3 covers microbiology and immunology. Each block culminates in an examination. The purpose of this block is to lay the groundwork for the systems to build upon. These courses are similar in content to courses that many pre-med students took in college, except for the vast volume of material that is covered in a short period.

These general principles are integrated into the systems. The first system is Cardiovascular-Blood, which is followed by Pulmonary and then Renal. Together, these three modules make up Block 2. There is an examination on each system at the end of its respective module. The final block of the first year is Musculoskeletal (MSK)-Dermatology, a six-week block that contains 4 weeks of MSK culminating in an anatomy practical exam, and 2 weeks of dermatology.

\section{The Initial Approach}

In college, I (CZ) majored in history of science, medicine, and public health, which is considered a humanities major. Alongside these courses, I took a pre-med course track along with other science courses. The workload was intense, but I was able to manage my priorities, as I still had time to be involved in extracurricular activities and performed well academically.

Like many other students, when I arrived in medical school, I started out with the strategies I used in college because the method had previously worked for me. I annotated PowerPoint slides during lecture, then retyped those notes and the PowerPoint information into a study guide based on the given learning objectives afterwards. While doing this, I would try to develop a strong understanding of the lecture.
In reviewing lectures, I went back to the guide and rewrote my notes out by hand over and over while highlighting anything I could not recall right away. The next time I went through the guide, I focused on those topics that I initially did not grasp, leading to more focused and efficient studying over time. By the time the exam came around, I would only have a few items to review. I also made use of Anki (a flashcard program that uses the method of spaced repetition to aid memorization) and created my own flashcard decks to help reinforce information.

\section{The Need to Change}

Although I was content with my performance in the Foundations block, I did notice that I was always studying and incredibly stressed, especially around examination time, which was rarely the case before medical school. Studies have shown that stress is higher in medical students compared to the general population and that it may have a higher impact in a medical student's first year compared to other years [8, 9]. Radcliffe and Lester showed that the number and intensity of examinations is a significant source of stress, alongside lifestyle changes, such as students living on their own, being in a new city, and the need for professional socialization [9]. Students with higher college GPA at admission often have higher stress levels, possibly due to pressure from personal or familial expectations [8]. Oftentimes, this occurs because students do not effectively use and/or monitor their study strategies [10], leading to an increased cognitive load, stress, and anxiety. For me, the significantly increased stress associated with school negatively impacted my enjoyment of learning. Like many first-year medical students, I was studying almost all the time, always felt behind, and, at times, even doubted if medical school was really for me [11].

High levels of stress also have implications for mental health and has been correlated with depression, which is more commonly seen in first-year medical students than third-year medical students, as well as burnout [8]. Burnout is defined as a trifecta of emotional exhaustion, depersonalization, and having a decreased sense of accomplishment [11, 12]. It affects almost $50 \%$ of medical students $[12,13]$. Students with burnout are more likely to seriously think about dropping out of medical school or consider suicide, which emphasizes the importance of preventing this phenomenon [11, 14].

Outside of health effects, stress also has a negative impact on learning and memory. While stress during encoding or just before it can boost memory formation, stress before learning can affect memory coding and reconsolidation [15].

Although my performance in the Foundations modules was satisfactory to me, I realized that some things needed to change for several reasons. First, my study approach would not be sustainable long-term. Second, my retention of 
information was not proportional to the effort I devoted to it. Third, and most importantly, learning was not as enjoyable anymore. As a result, I became interested in how humans learn and how to make my learning more efficient and effective. With the advisor, I was particularly interested in cognitive load theory (CLT) and dual processing theory, which have significant roles in medical diagnostics and medical education $[16,17]$.

\section{The Modified Approach Based on Learning Theories}

Cognitive load can be divided into three parts: intrinsic, extraneous, and germane [18]. Intrinsic cognitive load defines how complex the knowledge acquired is. If the learner's expertise and the difficulty level of the information given are already determined, then this load is essentially fixed. Extraneous cognitive load defines the methods used to present the material. Germane cognitive load is the component that increases when we create schemas to help us decrease our working memory load as it helps us consciously process information. The constructed schemas decrease the complexity of the information, thereby reducing intrinsic cognitive load, by taking multiple interacting elements and integrating them into a single concept, thus simplifying the information. This is the type of cognitive load that should be promoted while decreasing the other two types. However, it is important to note that intrinsic and extraneous cognitive load are additive and, given that we have a set amount of working memory, if the former two types of cognitive load are too high, there is not enough room to increase the germane cognitive load [19].

Dual processing theory is based on the idea that there are two systems that the brain uses to form thoughts: System 1 and system 2. System 1 thinking is faster, subconscious, and more effortless and system 2 is slower, analytical, and takes more effort $[17,20]$.

In the context of learning, the system 1 type of thinking is utilized when the learner starts to seek constructed patterns or schemas. For example, in learning the histology of the gastrointestinal system, the pattern of stratified vs. simple epithelium distribution correlates to their functions. Once the instructor demonstrates the importance of pattern recognition or the learners have sought the pattern, the effort needed for memorization is significantly reduced. Instead of memorizing the facts, learners can predict which type of epithelium would be present based on their location and function.

Illness scripts are another example of facilitating system 1 thinking [19]. These scripts typically contain a disease's epidemiology, presentation, pathophysiology, diagnosis criteria, and treatment [19]. They are a mental organization of the key features of diseases, which facilitates the prediction and estimates the likelihood of a diagnosis, depending on the presence or absence of those features [21-23]. Utilizing system 1 thinking is clearly important in developing expertise as a physician; however, it is also vital to the reduction of cognitive load within working memory during the preclinical years of medical school [20]. Constructing schema and increasing germane cognitive load are ways in which information taught in lectures can be better retained, remembered, and later utilized in system 1 thinking.

System 2 thinking is slower, more analytic, and conscious; it requires more effort than system 1 thinking [17]. This type of thinking is utilized when the findings do not fit in a pattern or schema and requires an understanding of the pathophysiology of a process to determine the likelihood of a diagnosis. For example, determining the cause of an acute kidney injury requires knowing the key features of a list of diagnosis candidates and their illness scripts, then working through the presentation, history, physical, and laboratory findings to create a list of differential diagnoses based on the likelihood.

With the realization that the overall efficiency and effectiveness of my study strategies needed improvement, I applied a modified, though similar, approach at the beginning of the system-based modules. However, because the information was much less familiar than that of Foundations, I found I needed double or triple the amount of time to watch and review each lecture. Because of the added effort it took to make the study guides for each lecture, as well as learn material unfamiliar to me, I had constant high levels of stress and anxiety during the first systems module.

In working with my advisor, I was able to examine my approach in depth and understand how and why it was no longer conducive to the learning of disease processes and clinical reasoning skills, as well as impacting knowledge retention. We were then able to integrate changes to my study strategies with the focus of decreasing cognitive load and promoting the schema seeking method.

First came more general changes to my note-taking and study methods: new organizational methods, schema seeking, and the selective use of ready-made Anki cards. The organizational methods mostly applied to note-taking. The first method was the creation of mind maps (Fig. 1) while watching lectures. Mind maps utilize visual diagrams' central themes and subcategories to help learners seek framework first, then organize and visualize information [24].

For learning and focusing on diseases, I used illness scripts to organize pathophysiology content in the system-based modules. This approach helps seek the main ideas and key features while listening to a lecture, making it easier to see patterns in real time. For example, using illness scripts for osteoarthritis and rheumatoid arthritis promoted pattern seeking and differentiation. Furthermore, the real-time notes become a customized study guide, eliminating that need to retype them after the fact. This method can make note-taking during lecture more engaging, reduce the amount of time 


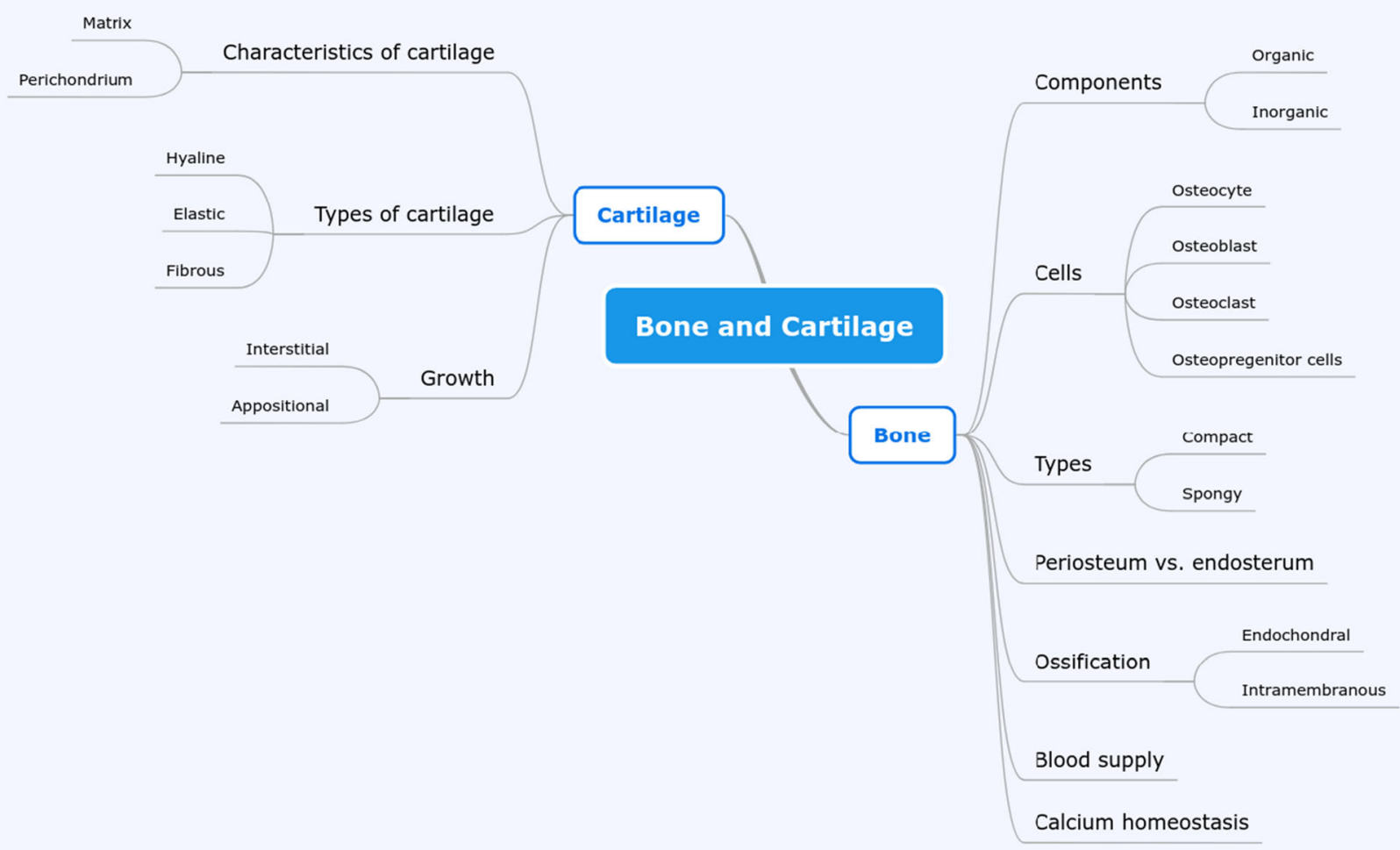

Fig. 1 A sample mind map

spent on organizing notes, and create clear goals for retention, which all serve to increase germane cognitive load. Consequentially, this reduces working memory load and helps improve system 1 thinking. It also reduces element interactivity, as this framework of an illness script condenses all the elements of a disease into a single visual presentation, as indicated by Van Merrienboer et al. [18, 19]. This method of schema searching and construction is important in future medical decision-making as a physician as well. Practicing this skill in the preclinical phase is essential in establishing a foundation in both knowledge and strategy [20].

The changing of study strategies was not met without resistance from within and it was uncomfortable at first. However, as a result of focusing on decreasing cognitive load and promoting schema seeking, learning in the system modules became more efficient, effective, and more enjoyable.

\section{Application of the Practical Approaches}

Two practical approaches were developed by the corresponding author. They are driven by the cognitive theory and aimed to create intentional, data-driven, and individualized ways to plan, self-assess, and learn. These approaches are Goals, Means, and Outcomes (GMO) and Self-assessment,
Identification, and Patching (SIP). They have been applied in the context of preparation for the United States Medical License Examinations (USMLE) in response to the widely canceled/rescheduled USMLE examinations in March-May of 2020 due to COVID [25].

Here we describe the GMO and SIP approaches to frame the efforts of the first-year students, regardless of an integrated, traditional, or problem-based curriculum. Setting up a goal is usually one of the first steps to study. However, knowing how to set up a goal that is attainable and has measurable outcomes when planning to study is often an afterthought. Therefore, we developed three practical questions to create the checkpoints for the process: "What is your goal?" "How are you going to achieve it?" and "How do you know that you have been successful?" The third question was designed to integrate SIP into GMO to better define the scope of the initial goal. Compare these two statements for setting a goal: "I would like to study the MSK system" vs. "I would like to study the immune component of MSK." The former goal includes all subjects from MSK, studied from top to bottom. This approach will take a long time to produce any tangible results. Furthermore, prolonged studying without any assessment is not conducive for students who are still struggling to find an effective study strategy, as it can lead to burnout [11, 26]. Dividing the general goal into "buckets," such as 
immunology in MSK, allows for a review of general principles of immunology, such as autoimmunity, as well as the application in specific diseases. It is also more manageable in scope.

With this goal set up, the next step is to choose the "Means" that can help attain the goal. The sources include lecture slides, cases, or illness scripts. Along with a number of commercially available resources, could be tailored to the student's learning modality preference. For example, using First Aid (an outline of high-yield topics organized by General Principles and Organ Systems) for United States Medical License Examination, students can look for the immunology content in the organ system of MSK, then specifically review the content in the section of general principles.

Finally, having a measurable "Outcome" demands a welldefined goal and accountability during the studying process, and provides an objective measurement of the effectiveness of the methods used. Currently, there are a limited number of commercially available questions for the foundational science of the first-year curriculum. Students can create questions under each of the learning objectives or use mind maps, to selfassess. Figure 1 shows a mind map from the Cartilage and Bone lecture learning objectives. The map displays the two major components of the lecture. Students can practice active recall (the first step of self-assessment) from the branches of the cartilage or the bone part of the map after the lecture, identify which components need patching, and continue the cycle of SIP. The results of the self-assessment are then used as directives for the "Identification" and "Patching." An intentional and effective self-assessment is one of the key elements and should result in the clear identification of the areas that need more attention.

Commercially available question banks have become a valuable learning and assessment tool [27]. The GMO method is especially important to be applied when using question banks to empower the students in the process of SIP. For example, if the first goal is "how am I doing in pathology and pathophysiology within the MSK system?" and the second goal is "how well have I integrated immunology general principles into MSK?" then two selections of questions defined the scope of self-assessment. The Outcome of the selfassessment allowed focused and timely identification and patching. Further, based on the missed questions, students can differentiate if there is a pattern of mistakes (e.g., major concepts vs. fact memorizations; content vs. test-taking). Then, they can focus their efforts in specifically patch the areas using the appropriate tools (e.g., if a major concept issue, targeted reading or watching lecture videos should be the next step, rather than continued use of questions). More importantly, students should reflect and modify their initial study strategy to prevent the continuation of creating such concept gaps. Because of the limited availability of questions for the firstyear students, students can expand the usage of the questions by rewriting the available question stem for each of the incorrect answer choices. This practice effectively helps differentiate the concepts or facts. The continued cycle of SIP offers focused exposure to the content and valid performance data.

Therefore, GMO and SIP are intertwined and continuous cycles for the intentional, data-driven and individualized learning. They can be applied while using learning objectives, framework of illness scripts, or flash cards and question banks.

\section{The Challenges of Learning During COVID-19}

As the time went on, for me (CZ), the gradual application of SIP and GMO helped decrease my stress levels and improve my retention, as well as helping me regain my love for learning.

However, the emergence of the COVID-19 pandemic posed new challenges. By March 2020, COVID-19 had significantly impacted every aspect of the country, including medical education. At Northwestern, the MSK system was taught virtually. As a part of this, classes were mostly taught via pre-recorded lectures or over Zoom. Many in-person clinical skills sessions, such as learning the MSK exam, were pushed to M2 year. A big roadblock the module leaders had to overcome for this particular system was figuring out how to do anatomy labs virtually. Northwestern purchased a subscription to Complete Anatomy for its students and recorded lectures with photographs of dissections to help us better be able to visualize muscles without seeing a cadaver in person. Still, it was a different way of learning compared to past anatomy labs and it took a little time to get used to as a student.

I personally found the biggest challenge of a virtual curriculum to be the lack of structure that came with moving classes online. I did not need to get up for an 8 a.m. class, had no library to study in with friends between classes and small groups, and was essentially setting my own schedule. It was made even more difficult because before the pandemic, I relied on doing activities such as grocery shopping or getting lunch with friends to break up studying into smaller chunks over the course of a module. Suddenly, I was at home in the same environment all day with nothing to break up the monotony. Because of that, it was evident early on that it would be very easy for me to lose focus or get behind.

As a solution, I made my own schedule to simulate a regular school day, making sure to keep GMO in mind. I always set a certain number of lectures I wanted to watch each day and continuously assessed myself using the learning objectives. I also picked up new hobbies and scheduled in breaks as an incentive to focus on the GMO of the day. Each week, I scheduled time to practice SIP to patch up what I had identified as weaker topics using either the learning objectives or a question bank. With the continuation of being mindful of 
cognitive load, seeking schema, and applying GMO and SIP, I was able to complete the first year of medical school with the renewed confidence and enjoyment of learning despite the challenge of virtual learning.

\section{Takeaway Message}

The demands of medical school, especially at the beginning, can be overwhelming physically, mentally, and emotionally. Monitoring data and outcomes (performance and wellbeing) when using past study habits and adapting new, more efficient strategies may help students' confidence and promote their wellbeing.

The practical approaches of SIP and GMO can be applied at various learning levels or academic situations. Taking the time to think about the outcome of a seemingly challenging lecture or topic may enable students to seek feasible goals and be rewarded with success sooner. The repeated SIP cycle provides a focused and accountable approach for a tailored process. By GMO and SIP together, the learning process will be intentional, data-driven, and individualized for each student.

\section{Compliance with Ethical Standards}

Conflict of Interest The authors declare that they have no conflict of interest.

Ethical Approval NA

Informed Consent NA

\section{References}

1. Lujan HL, DiCarlo SE. First-year medical students prefer multiple learning styles. Adv Physiol Educ. 2006;30(1):13-6.

2. Andriole DA, Jeffe DB. Prematriculation variables associated with suboptimal outcomes for the 1994-1999 cohort of US medical school matriculants. Jama. 2010;304(11):1212-9.

3. Huff KL, Fang D. When are students most at risk of encountering academic difficulty? A study of the 1992 matriculants to U.S. medical schools. Acad Med. 1999;74(4):454-60.

4. Kleshinski J, Khuder SA, Shapiro JI, Gold JP. Impact of preadmission variables on USMLE step 1 and step 2 performance. Adv Health Sci Educ Theory Pract. 2009;14(1):69-78.

5. Bierer, S.B. and E.F. Dannefer, The learning environment counts: longitudinal qualitative analysis of study strategies adopted by firstyear medical students in a competency-based educational program. Acad Med. 2016;91(11 Association of American Medical Colleges Learn Serve Lead: Proceedings of the 55th Annual Research in Medical Education Sessions):S44-s52.
6. Simanton E, Hansen L. Long-term retention of information across the undergraduate medical school curriculum. S D Med. 2012;65(7):261-3.

7. Heiman HL, O'Brien CL, Curry RH, Green MM, Baker JF, Kushner RF, et al. Description and early outcomes of a comprehensive curriculum redesign at the Northwestern University Feinberg School of Medicine. Acad Med. 2018;93(4):593-9.

8. Nechita F, Nechita D, Pîrlog MC, Rogoveanu I. Stress in medical students. Romanian J Morphol Embryol. 2014;55(3 Suppl):12636.

9. Radcliffe C, Lester H. Perceived stress during undergraduate medical training: a qualitative study. Med Educ. 2003;37(1):32-8.

10. Boyer SL, et al. Self-directed learning: a tool for lifelong learning. J Mark Educ. 2013;36(1):20-32.

11. Ishak W, et al. Burnout in medical students: a systematic review. Clin Teach. 2013;10(4):242-5.

12. Frajerman A, Morvan Y, Krebs MO, Gorwood P, Chaumette B. Burnout in medical students before residency: a systematic review and meta-analysis. Eur Psychiatry. 2019;55:36-42.

13. Dyrbye LN, Sciolla AF, Dekhtyar M, Rajasekaran S, Allgood JA, Rea M, et al. Medical school strategies to address student wellbeing: a national survey. Acad Med. 2019;94(6):861-8.

14. Dyrbye L, Shanafelt T. A narrative review on burnout experienced by medical students and residents. Med Educ. 2016;50(1):132-49.

15. Sandi C. Stress and cognition. Wiley Interdiscip Rev Cogn Sci. 2013;4(3):245-61.

16. Young JQ, van Merrienboer J, Durning S, ten Cate O. Cognitive load theory: implications for medical education: AMEE Guide No. 86. Med Teach. 2014;36(5):371-84.

17. Kahneman D. Think, fast and slow, vol. 499. New York: Farrar, Straus and Giroux; 2010.

18. Sweller J, Merrienboer J, Pass F. Cognitive architecture and instructional design. Ed Psych Rev. 1998;10(3):251-96.

19. van Merrienboer JJ, Sweller J. Cognitive load theory in health professional education: design principles and strategies. Med Educ. 2010;44(1):85-93.

20. Trowbridge R, Rencic J, Durning S. Teaching clinical reasoning. United States: American College of Physicians; 2015. p. 270.

21. Bowen JL. Educational strategies to promote clinical diagnostic reasoning. N Engl J Med. 2006;355(21):2217-25.

22. Charlin B, Boshuizen HPA, Custers EJ, Feltovich PJ. Scripts and clinical reasoning. Med Educ. 2007;41(12):1178-84.

23. Custers EJ. Thirty years of illness scripts: theoretical origins and practical applications. Med Teach. 2015;37(5):457-62.

24. Phenwan T, Tawanwongsri W. Retained mind mapping skills and learning outcomes in medical students: a mixed methods study. MedEdPublish. 2018;7(4).

25. Brianna Borsheim CL, Zitelny E, Zhao C, Blizzard J, Hu Y. Preparation for the United States Medical Licensing Examinations in the face of COVID-19: Medical Science Educator; 2020.

26. Dyrbye LN, Thomas MR, Power DV, Durning S, Moutier C, Massie FS Jr, et al. Burnout and serious thoughts of dropping out of medical school: a multi-institutional study. Acad Med. 2010;85(1):94-102.

27. Banos JH, Pepin ME, Van Wagoner N. Class-wide access to a commercial step 1 question bank during preclinical organ-based modules: a pilot project. Acad Med. 2018;93(3):486-90.

Publisher's Note Springer Nature remains neutral with regard to jurisdictional claims in published maps and institutional affiliations. 